\title{
Archéopages
}

Archéopages

Archéologie et société

42 | 04-07/2015

Construire en terre crue

\section{Les enduits de terre crue de deux fosses antiques}

Recherches pluridisciplinaires et hypothèses de fonctionnement

Two Roman clay-lined pits. Multi-disciplinary analyses and hypotheses

concerning their use

Las argamasas de barro de dos fosas antiguas. Investigaciones

pluridisciplinarias e hipótesis de funcionamiento

Tanguy Wibaut, Jean-Emmanuel Aubert, Jérôme Ros, Jérôme Kotarba et Pascal Verdin

\section{(2) OpenEdition}

\section{Journals}

Édition électronique

URL : https://journals.openedition.org/archeopages/1248

DOI : 10.4000/archeopages. 1248

ISSN : 2269-9872

Éditeur

INRAP - Institut national de recherches archéologiques préventives

Édition imprimée

Date de publication : 1 mars 2016

Pagination : 88-93

ISSN : 1622-8545

Référence électronique

Tanguy Wibaut, Jean-Emmanuel Aubert, Jérôme Ros, Jérôme Kotarba et Pascal Verdin, « Les enduits de terre crue de deux fosses antiques », Archéopages [En ligne], 42 | 04-07/2015, mis en ligne le 01 juillet 2017, consulté le 03 juin 2021. URL : http://journals.openedition.org/archeopages/1248 ; DOI : https://doi.org/10.4000/archeopages.1248 


\title{
Les enduits de terre crue de deux fosses antiques Recherches pluridisciplinaires et hypothèses de fonctionnement
}

\author{
Tanguy Wibaut ${ }_{\text {Irrap }}$ \\ avec la collaboration de Jean-Emmanuel Aubert universitéde Toulouse, Laboratoire Matériaux et Durabilité des Constructions (LMDC) \\ JérÔme ROS CNRS, UMR 7209, «Archéozoologie, Archéobotanique: sociétés, pratiquese et environnements»" \\ Jérôme Kotarba Inrap \\ Pascal Verdin Inrap, UMR 7264, «CEPAM»
}

\section{8}

La ville de Prades (Pyrénées-Orientales) se situe dans la moyenne de vallée de la Têt, dans les premiers contreforts des Pyrénées orientales. Une opération de fouille ${ }^{\mathbf{1}}$, implantée en périphérie immédiate de l'ancienne ville médiévale, a révélé des vestiges datés entre le $\mathrm{I}^{\mathrm{er}}$ siècle et le $\mathrm{v}^{\mathrm{e}}$ de notre ère, liés à un habitat antique dont la localisation précise et l'ampleur restent incertaines. La documentation actuelle ne permet donc pas de préciser la nature et le statut exact de ce site. Deux fosses (SB1 au nord et SB65 au sud, distantes l'une de l'autre d'environ $9 \mathrm{~m}$ ) comblées au cours de la seconde moitié du i ${ }^{\text {er }}$ siècle, entre 50 et 80 de notre ère, ont particulièrement attiré l'attention du fait de leur revêtement [ill. 1].

\section{Des fosses pourvues d'un revêtement en terre crue}

Les deux fosses apparaissent à $40 \mathrm{~cm}$ de profondeur sous le niveau actuel. Leurs limites se distinguent bien du sol naturel environnant et particulièrement le liseré de terre plus claire qui tapisse les parois du creusement [ill. 2]. En plan, chacune d'elles dessine une forme rectangulaire à angle arrondi. Elles présentent des dimensions comparables et un volume maximal de $10 \mathrm{~m}^{3}$. Elles sont creusées dans des niveaux naturels graveleux. Les parois sont très légèrement convexes et le fond est aplani bien qu'en pente vers une extrémité [ill. 3]. Ces fosses se singularisent par la présence d'un épais revêtement constitué de terre crue, de $6 \mathrm{~cm}$ au fond et jusqu'à $22 \mathrm{~cm}$ en haut des parois, qui s'épaissit au niveau du fond de la fosse nord. Sa pose réalisée de façon soignée a permis de ragréer le modelé du terrain naturel, irrégulier par endroits. Notons qu'une partie du fond de la fosse sud n'a pas été recouverte par cet enduit, profitant de la présence à cet emplacement d'une strate de limon jaune naturel. La surface de l'enduit est lisse et dessine une légère courbure à la jonction entre le fond et la paroi. Globalement, cet enduit est d'aspect homogène. Le sédiment qui le compose est compact et de couleur jaune pâle, il est toutefois plus beige dans la partie basse de la fosse nord. La matrice limoneuse inclut quelques charbons de bois, de rares galets et des cailloux épars. Telles qu'elles nous sont parvenues, ces fosses sont dépourvues de système d'accès. La dynamique de comblement est identique pour les deux fosses. Le remblaiement se compose de plusieurs strates limoneuses, plus ou moins foncées et/ou sableuses, et il comporte par endroits des passées de terre charbonneuse, correspondant à des vidanges de foyer, de rares scories de fer ainsi que des blocs dont certains forment des concentrations. Ces couches 


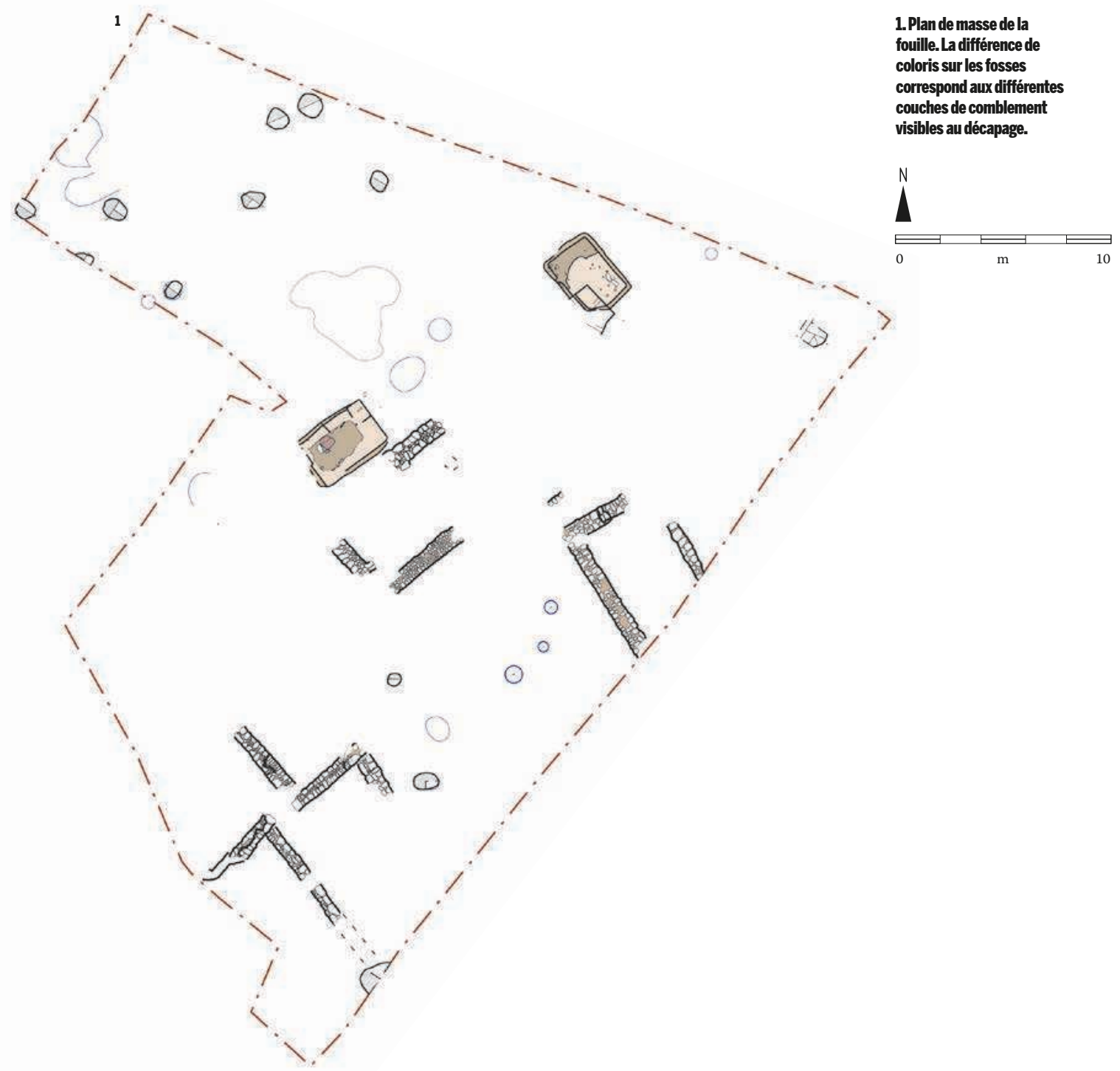

1.Plan de masse de la

coloris sur les fosses

correspond aux différentes

couches de comblement

visibles au décapage.

2. La fosse nord, testée dans son quart sud-est lors du diagnostic. On

distingue le parement de terre crue des parois et l'enduit plus clair du fond.

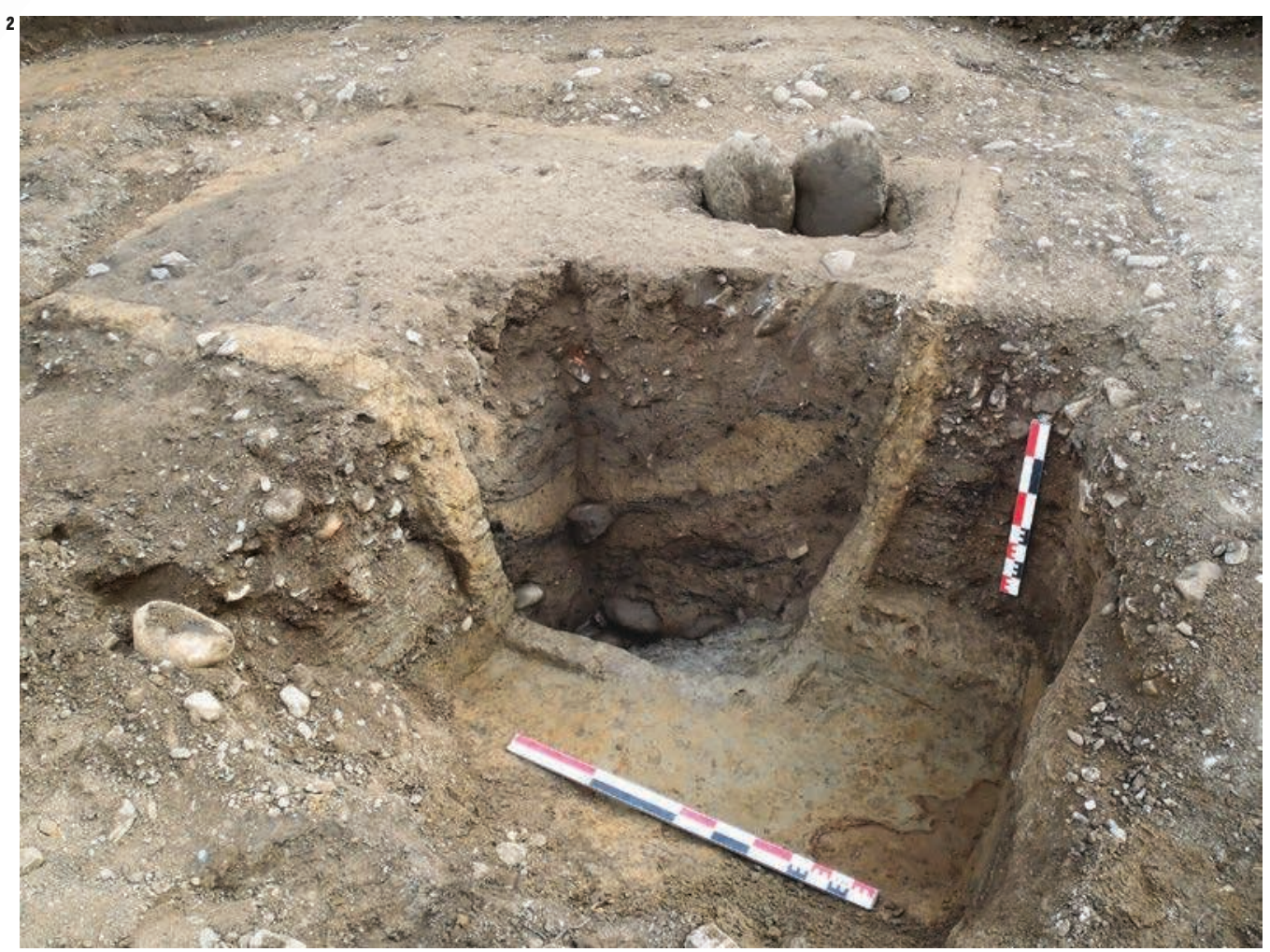


3. Coupes latérales et longitudinales des deux

fosses. Elles présentent

des dimensions similaires,

environ 2,20 $\mathrm{m}$ de large

pour 3,20 m de long ; seul

la profondeur diffeère,

jusqu'à $1,40 \mathrm{~m}$ pour la

fosse nord et seulement

$1,10 \mathrm{~m}$ pour l'autre dont

le fond correspond sur

une large moitié nord

à la couche naturelle

limoneuse.
3

\section{La fosse nord}

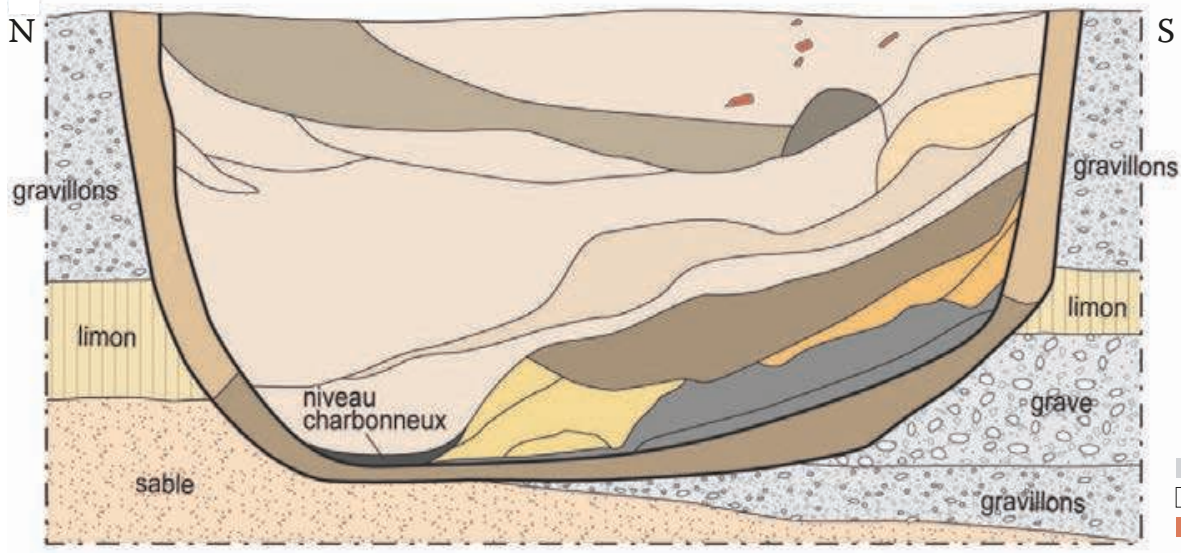

Scorie $\checkmark$ Pierre Céramique

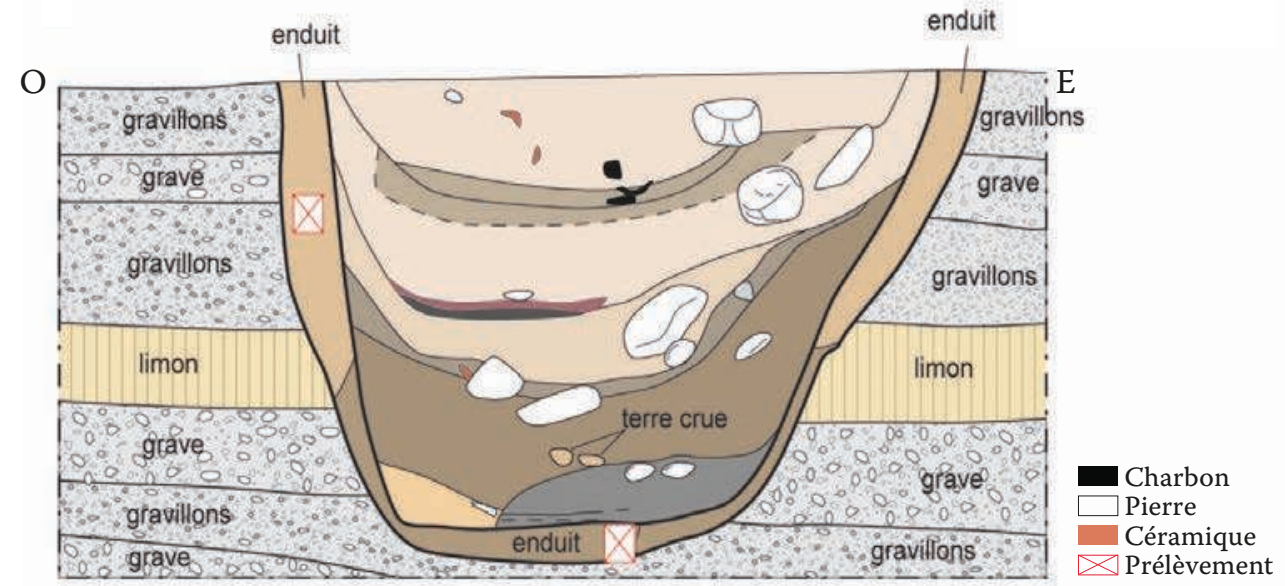

\section{La fosse sud}
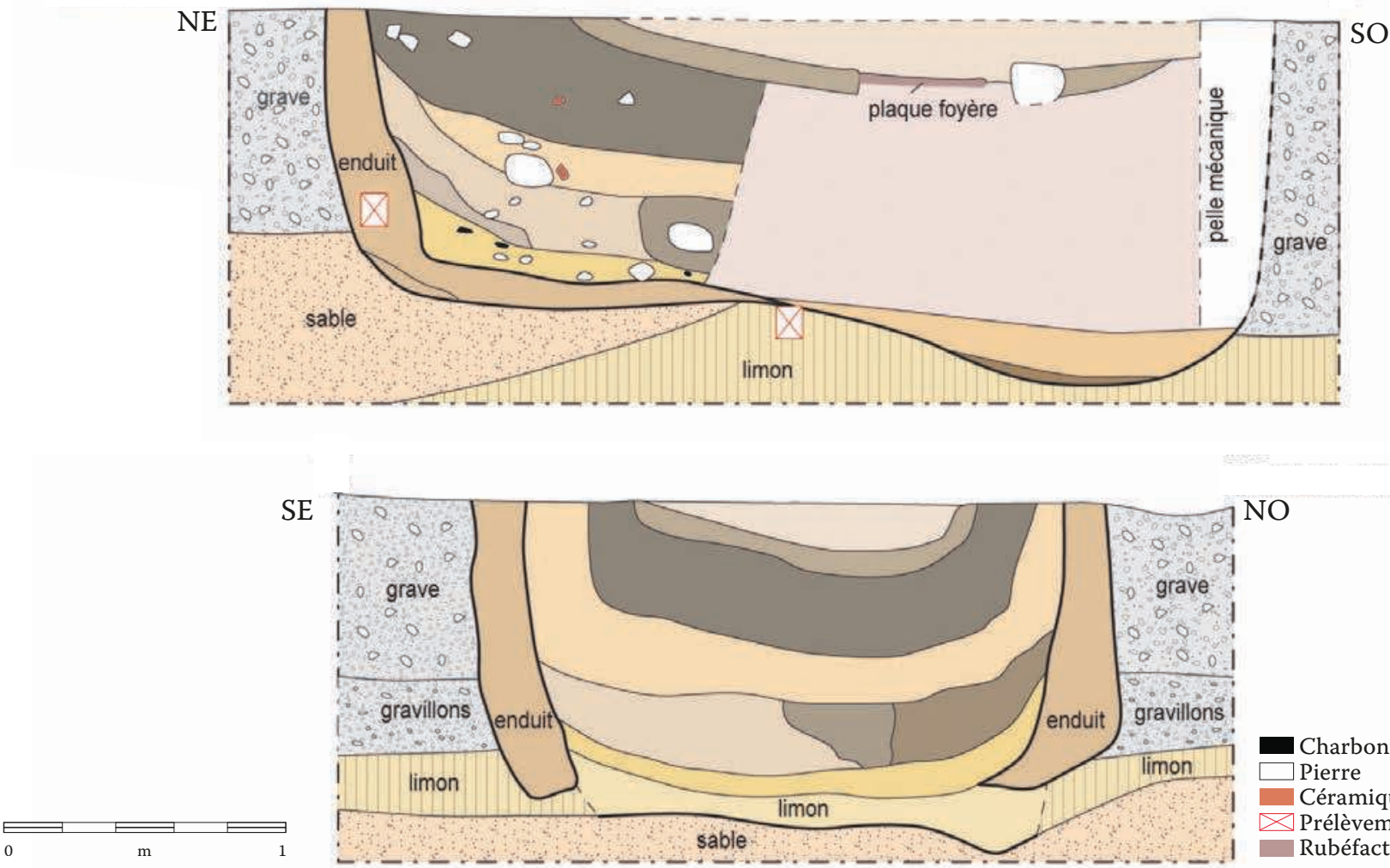

$\mathrm{NO}$

Charbon

Pierre

Céramique

$\varangle$ Prélèvement Rubéfaction 
remontent le long des parois latérales, d'où un profil en U évasé, dû à des tassements distincts. Par contre, longitudinalement, les niveaux inférieurs épousent le fond de la fosse qui présente un léger pendage.

\section{Un revêtement limoneux d'extraction locale}

Des analyses physico-chimiques et minéralogiques effectuées sur trois échantillons - provenant des parois des deux fosses, du fond de la fosse nord et de la couche naturelle limoneuse atteinte lors du creusement de la fosse sud [ill. 4c] (Thouron, 2015) ${ }^{2}$ - ont contribué à l'interprétation du rôle joué par l'enduit. Ces analyses permettent en effet d'identifier la nature des terres utilisées et la composition du mélange nécessaire à la confection de l'enduit.

La caractérisation physique a pour but de mettre en évidence d'éventuelles différences granulométriques ${ }^{3}$ entre le limon naturel et les revêtements situés sur le fond et les murs, en distinguant différentes classes granulométriques (argiles, limons, sables, graviers et cailloux). Les résultats semblent indiquer que les enduits ont une origine commune [ill. 4a], sans doute un matériau proche du limon naturel, présent sur le site à $80 \mathrm{~cm}$ de profondeur. Nous observons néanmoins que la teneur en argile varie quelque peu d'un échantillon à l'autre : le fond de la fosse nord présente un taux de $25 \%$ de particules très fines alors que celui des parois est légèrement inférieur, de l'ordre de $15 \%$. Si ce taux est trop faible pour que l'enduit soit étanche à l'eau, ce dernier peut toutefois réguler l'hygrométrie à l'intérieur de la fosse et de la sorte préserver son éventuel contenu des risques liés à l'humidité (pourrissement...).

L'analyse chimique ${ }^{\mathbf{4}}$ permet quant à elle de préciser la composition de cet enduit. Elle révèle que les quatre échantillons sont constitués des éléments majeurs suivants : aluminium, silicium, fer, magnésium, manganèse, calcium, sodium, potassium, titane et phosphore. Chaque élément

2. L'analyse des

échantillons est le fruit

d'une collaboration

scientifique entre l'Inrap

et J.-E. Aubert du

Laboratoire Matériaux et

Durabilité des

Constructions (LMDC)

de l'Université de

Toulouse. Ces

investigations ont été

gracieusement prises en

charge par le laboratoire

dans le cadre du stage de

Mélissa Thouron.

3. Les tests sont réalisés

par tamisage à sec pour

les particules supérieures

à $80 \mu \mathrm{m}$ et par

sédimentation pour les

particules inférieures à

$80 \mu \mathrm{m}$. La sédimentométrie

détermine la distribution

pondérale de la taille des

\begin{tabular}{|c|c|}
\hline & \\
\hline & fur et \\
\hline & température de \\
\hline érenciées & l'échantillon augmente \\
\hline par cette méthode. & $\left(\right.$ de $\left.20^{\circ} \mathrm{Cà} 1000^{\circ} \mathrm{C}\right)$ \\
\hline 4. Il s'agit d'une analyse & Les variations de masse \\
\hline avec une ICP-OES & à certaines températures \\
\hline (Induced Coupled Plasma & sont caractéristiques de la \\
\hline Optical Emission & transformation de certains \\
\hline Spectroscopy) qui a été & minéra \\
\hline menée par le Service & xemple, \\
\hline d'Analyse des Roches et & à $120^{\circ} \mathrm{C}$, la pert \\
\hline des Minéraux (SARM) & \\
\hline du Centre de Recherches & de l'eau hygroscopique. \\
\hline Pétrographique & À $500{ }^{\circ} \mathrm{C}$, c'est la \\
\hline RPG) de & déshydratation des argiles. \\
\hline s remercions & 6. La goethite est un \\
\hline vivement ces organismes & oxyhydroxyde de fer. \\
\hline pour leur collaboration & 7. Analyses réalisées par \\
\hline qui a permis, dans ce cadre & Cécilia Cammas, Inrap. \\
\hline spécifique, d’avoir recours & 8. Analyses réalisées par \\
\hline à des analyses inhabituelles. & Pascal Verdin, Inrap. \\
\hline 5. Durant l'analyse, la & 9. Étude réalisée par \\
\hline & Jérôme Ros, CNRS. \\
\hline
\end{tabular}

est représenté dans des proportions similaires d'un échantillon à l'autre [ill. 4b]. On s'aperçoit toutefois qu'il y a $4 \%$ de moins de dioxyde de silicium dans le limon naturel que dans les autres échantillons. Les enduits ont donc probablement été mélangés avec du sable de rivière. Le revêtement des parois étant plus épais que le fond, l'adjonction de sable a permis de limiter, voire d'éviter la fissuration de l'enduit au moment du séchage.

La caractérisation minéralogique a permis de confirmer la provenance locale des matériaux. Premièrement, une analyse menée par diffraction de rayons $\mathrm{X}(\mathrm{DRX})$ a été réalisée pour identifier la nature des minéraux cristallisés majeurs présents dans chaque échantillon. Ceux-ci contiennent de la montmorillonite (composante des smectites), de l'illite (le minéral le plus commun dans les argiles), de la muscovite (c'est-àdire du mica blanc), de l'orthose, de l'albite et des cristaux de quartz. La signature minéralogique est caractéristique du massif du Canigou. La présence de mica dans le limon est de nature à renforcer la texture de l'enduit à l'état sec. Deuxièmement, une analyse thermique gravimétrique (ATG) a été réalisée $e^{\mathbf{5}}$. Ce test a permis de compléter les analyses faites par DRX en mettant notamment en évidence l'absence de goethite ${ }^{\mathbf{6}}$ et la présence d'hydrargillite dans l'enduit du fond de la fosse nord. Inversement, dans les autres échantillons, on constate la présence de goethite et l'absence d'hydrargillite. L'absence de goethite expliquerait le moindre taux en oxyde de fer et démontre également que le matériau servant de base à l'enduit qui tapisse le fond de la fosse nord, même s'il est apparenté au limon naturel par sa composition chimique et sa granulométrie, a pu être prélevé à un autre endroit.

Des analyses micromorphologiques, actuellement en cours d'étude ${ }^{7}$, devraient quant à elles permettre de caractériser la mise en ouvre utilisée pour l'application des enduits.

\section{L'apport des macrorestes}

Si ces analyses ont permis de mieux cerner l'origine des matériaux qui enduisent les fosses, elles n'ont toutefois qu'un intérêt limité pour appréhender la fonction initiale de celles-ci. Nous avons donc complété ces premières investigations par la recherche de phytolithes ${ }^{\mathbf{8}}$. Un prélèvement extrait dans l'enduit du fond de la fosse nord a livré la présence de phytolithes de graminées $(89,1 \%)$ et de phytolithes de taxons ligneux (arbres soit 10,9\%). Parmi les phytolithes de graminées, on soulignera la présence de glumes de graminées sauvages $(11,9 \%)$ et de glumes issus plus probablement d'orge (type Hordeum, 4,5 \%) et de blé (type Triticum, o,5\%).

Un second échantillon de sédiment, prélevé dans la même couche et destiné à l'étude des carporestes ${ }^{\mathbf{9}}$, n'a pas permis de mettre en évidence un assemblage en position primaire. En effet, les semences identifiées correspondent à des éléments 


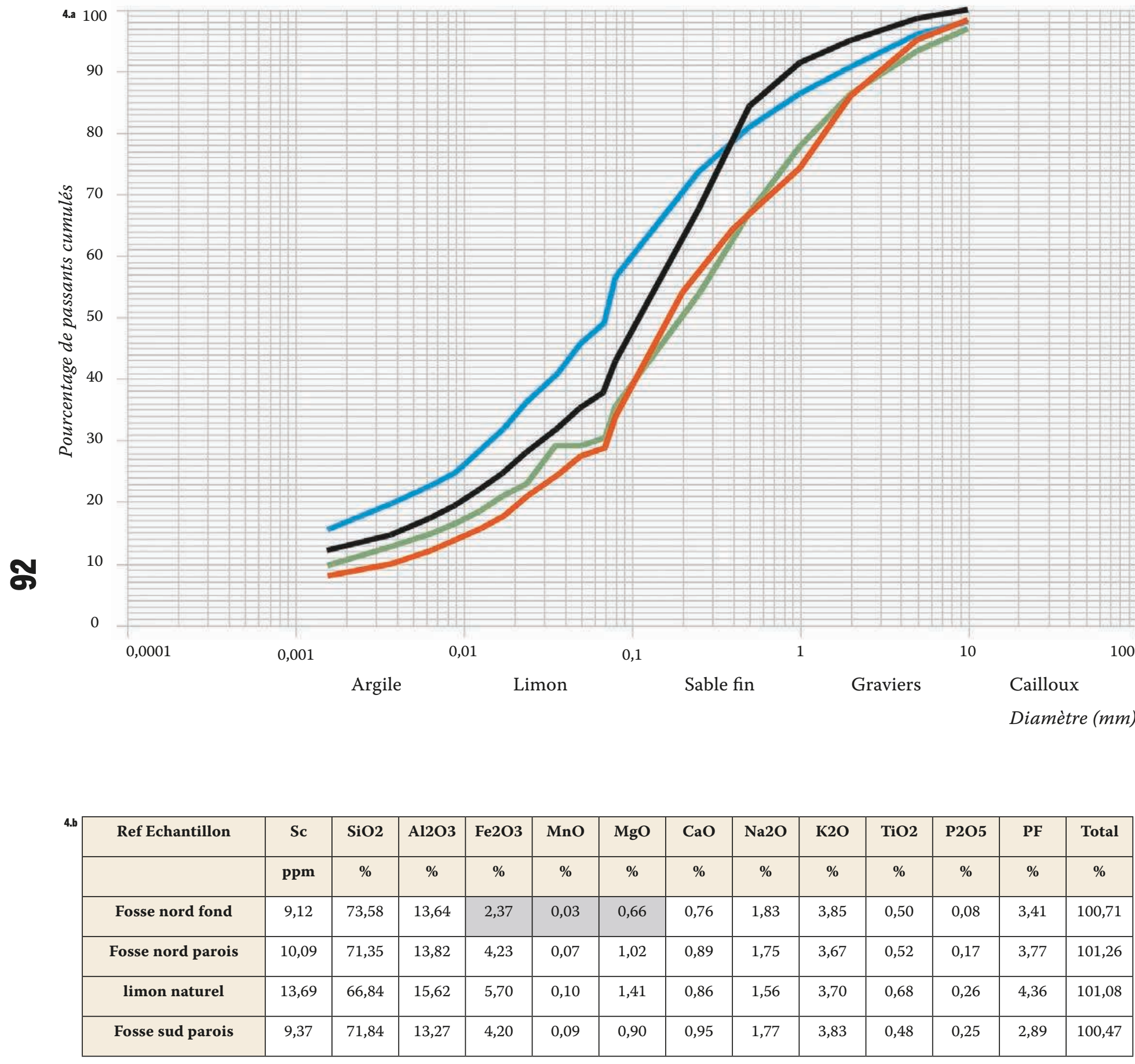

4.a. Courbes granulosédimentométriques des des éléments majeurs le limon naturel du fond de présents dans les quatre la fosse sud, en vert échantillons (SB1 est la l'enduit de ses parois. Le fosse nord; SB65, la fosse bleu et le rouge sud). L'enduit du fond de 1 correspondent fosse nord comporte respectivement au fond et moins d'oxyde de fer à l'enduit des parois de la $\quad$ (Fe203) et de manganèse fosse nord.

\section{(MnO) que les autres} enduits, ce qui explique sans doute sa coloration plus claire. 
10. Dans les Pyrénées

Orientales et notamment dans la plaine côtière,

le recours à l'ensilage perdure durant toute

l'époque romaine. $\mathrm{Au}$ milieu du ${ }^{\text {er }}$ siècle de notre ère, l'utilisation de structures d'un volum proche de $15 \mathrm{~m}^{3}$ est associée, de manière intuitive, à des conservations particulières comme celle du fourrage.

11. Les morceaux de tegulae du comblement, certes postérieur à l'usage premier, sont très peu nombreux et ne représentent au total pas plus d'une demi-tuile. 12. Elles sont mieux documentées ailleurs en France et souvent considérées comme des pièces souterraines. d'origine détritique, rejets de consommation ou de préparation alimentaire, carbonisés puis rejetés dans la fosse. L'absence de restes carpologiques en position primaire de stockage ne signifie cependant pas que la fosse n'a pas servi de structure de réserve, les semences ne se conservant en place qu'en cas de combustion accidentelle du stock ou lors du nettoyage des parois au feu pour assainissement.

\section{La fonction des fosses}

Des scories de fer, liées à la réduction du minerai, et des rejets de foyers sont attestés dans le comblement mais, bien entendu, ces vestiges ne peuvent pas illustrer la première utilisation de ces fosses. La question de la fonction de l'enduit peut être posée : il peut avoir servi soit à assainir la fosse, pour que le contenu reste au sec, soit à éviter des écroulements du substrat par ailleurs très friable. On pense donc à une fonction proche de celle d'un silo ${ }^{\mathbf{1 0}}$, même si les analyses et la morphologie atypique de ces deux fosses ne permettent pas de définir précisément leur fonction initiale. La perméabilité du revêtement en terre des parois et d'une partie du fond implique soit une couverture pour rester à l'air libre, soit un contact avec la matière à conserver et un recouvrement léger. L'hypothèse de simples caves n'a pas été retenue du fait de plusieurs éléments : l'absence d'aménagement pour descendre, l'absence de signes manifestes de piétinement sur le fond associé à une dégradation progressive des parois et enfin l'absence d'aménagement périphérique qui aurait montré l'intégration des fosses à un bâti assurant leur couverture ${ }^{\mathbf{1 1}}$. Comme le suggère timidement l'analyse des phytolithes, ces creusements pourraient avoir servi à la « réserve » de végétaux, comme par exemple du fourrage (Gazenkeek et al., 2015). Il est intéressant de rappeler dans ce cadre que les agronomes hispanoarabes préconisent, pour assainir les silos, l'utilisation « d'enduit composé d'argile, d'eau, d'olive et de cendres (d'autres composantes peuvent être ajoutées : coloquinte, myrte séchée, cyprès...)»(Puig, 2003, p. 112).

La forme quadrangulaire de ces fosses, inhabituelle dans la région ${ }^{\mathbf{1 2}}$, pourrait être liée à la difficulté de pratiquer un creusement en forme d'ampoule dans un terrain naturel instable. Ces fosses participeraient ainsi des activités agricoles de l'habitat tout proche, et elles pourraient en particulier jouer un rôle dans le domaine de la conservation des denrées, par exemple pour le bétail.

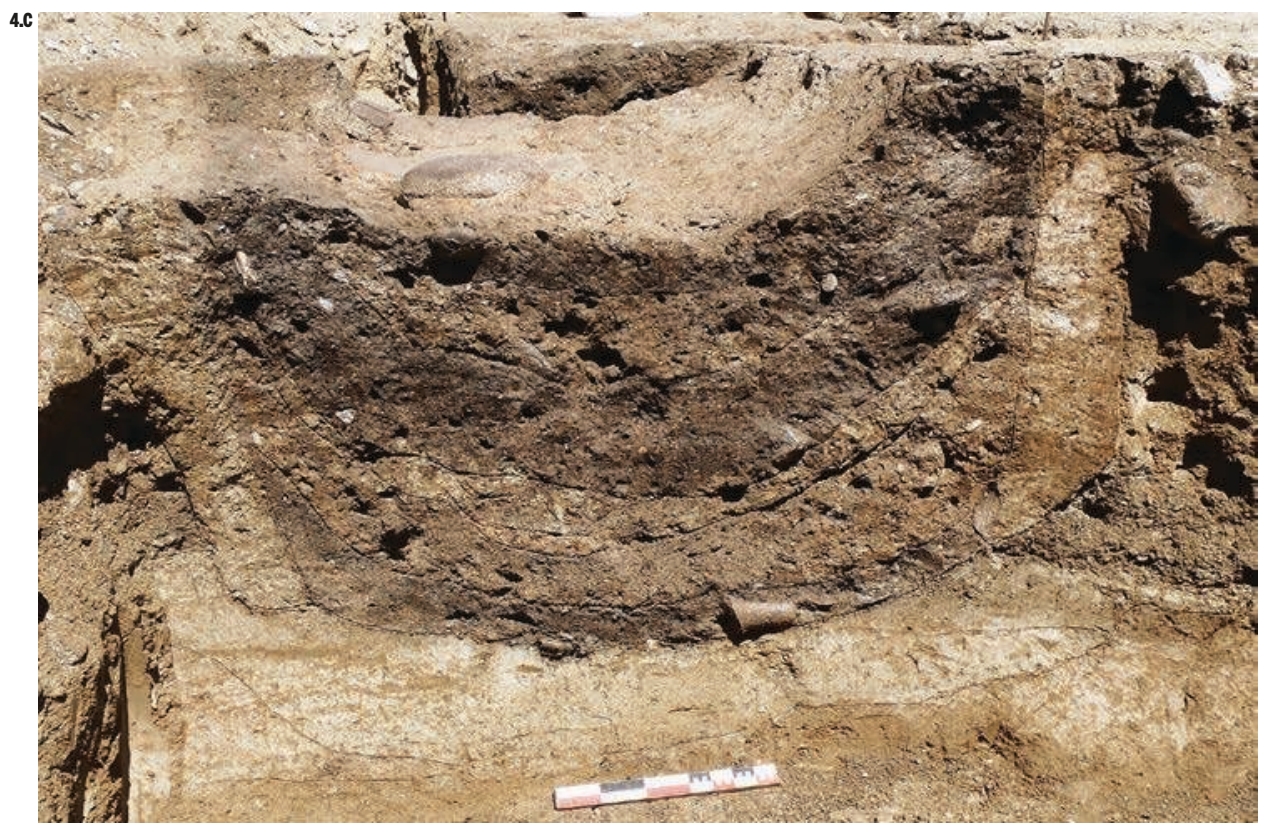

4.c. Vue en coupe de la

fosse sud où l'on observe

les parois de terre crue

posées sur le limon naturel

faisant office de fond.

\section{Références bibliographiques}

Gazenbeek M., Wiethold J., Verdin P., 2015, « Eine Domus in einer römischen Provinzstadt: La Fontainotte in Grand (Vosges) - archäologische und archäobotanische Ergebnisse der Ausgrabung 2011 ", in Косн M. (ÉD.), Archäologie in der Grossregion. Beiträge des internationalen Symposiums zur Archäologie in der Großregion in der Europäischen Akademie Otzenhausen, Archäologietage Otzenhausen, 1, p. 233-256.

Puig C., 2003, « Stockage et conservation des denrées agricoles en Roussillon du XI ${ }^{\mathrm{e}}$ au XIV ${ }^{\mathrm{e}}$ siècle », in Elne ville et territoire, Deuxièmes rencontres d'histoire et d'archéologie d'Elne (octobre 1999), Elne, Société des Amis d'Illibéris, p. 105-117.

Thouron M., 2015, Analyse minéralogique de parois en terre crue de vestiges archéologiques à Prades (66), rapport de stage Master 1 « eaux, sols, environnement » effectué sous la direction de J.-E. Aubert au laboratoire Matériaux et Durabilité des Constructions (UPS-INSA Génie Civil), Université de Toulouse, $25 \mathrm{p}$. 